We used data from National TB Program and included all cases of TB diagnosed in the Albania from 2010 to 2016. Information on age, sex, year of diagnosis, and anatomic location of the site of disease was retrieved from central database of National TB Program.

In Albania during 2010-2016, 925 cases of extrapulmonary TB were reported, males were $581(63 \%)$ and females 344 (37\%). The number of cases diagnosed per year was as follows: $170(38.2 \%)$ in 2010, $129(30 \%)$ in 2011, 108 $(25.7 \%)$ in $2012,141(29.7 \%)$ in $2013,147(36 \%)$ in 2014 , $117(28.2 \%)$ in 2015 and $113(27.2 \%)$ in 2016.

Sputum smear examination, X-ray and culture examination and tissue biopsy were carried out in 58; 42.3 ; 18 and $15 \%$ of patients respectively for EPTB diagnosis. The most affected age group was $<65$ years $(23 \%)$. Pleural effusion (35\%) and lymph node (15.7\%) were the most common types of extrapulmonary TB.

Patients live in urban areas $(60 \%)$ rather than rural (40\%). The mean age of EPTB patients is 44.5 and pulmonary TB patients is 41.2. Incidence of EPTB decreased from 5.5/100 000 in 2010 to 5.1/100 000 in 2016.

In Albania, extrapulmonary TB in 2010-2016 showed a slight decrease in incidence, although the rates are still very high. Diagnosis of extrapulmonary TB was made according to national guidelines, however long delay has been reported in most cases before the final diagnosis. Microbiological proof is the key to diagnosis and treatment, and tissue biopsy that should be required regularly.

\section{DETERMINANTS OF TB RELATED DEATH FROM TUBERCULOSIS PATIENTS IN THE NORTHERN THAILAND}

R. Miyahara' ${ }^{1}$ H. Yanai ${ }^{2}$, S. Mahasirimongkol ${ }^{3}$, L. Toyo-Oka ${ }^{1}$, K. Tokunaga ${ }^{1}$

${ }^{1}$ Department of Human Genetics, Graduate School of Medicine, The University of Tokyo, Japan; ${ }^{2}$ Fukujuji Hospital,

Japan Anti-Tuberculosis Association, Kiyose, Japan;

${ }^{3}$ Medical Genetics Center, Medical Life Sciences Institute,

Department of Medical Sciences, Ministry of Public Health,

Nonthaburi, Thailand

Tuberculosis is the most common cause of deaths from respiratory infection in Thailand. Understanding the risk of death could provide useful information to provide better clinical care for TB patients. $N$-Acetyltransferase 2 (NAT2) gene is the main determinant of isoniazid (INH) metabolism. NAT2 rapid acetylator contributes to lower anti-TB drug (INH) serum concentration and increased risk of treatment failure and relapse from INH based TB regimens.

The aim of this study was to determine the effect of NAT2 acetylator status on TB related death.

TB patients were recruited from the TB registry during 2002-2011 in Chiang Rai province, Thailand. The NAT2 acetylators (rapid, intermediate and slow) were determined by haplotype specific polymerase chain reactions, HS-PCR. These groups of patients were excluded from further analysis: 1) patients who did not receive the INH based regimens for TB treatment; 2) patients who did not receive the INH based regimens longer than 2 weeks and 3) patients who died within the first 2 weeks of TB treatment. Mortality-associated risk factors within 1 year of treatment were analyzed using Coxregression model.

Of 1.076 TB patients who met study criteria, 213 (19.8\%), $495(46.0 \%)$ and $368(34.2 \%)$ belonged to NAT2 rapid, intermediate and slow acetylate group respectively.
In total, 115 patients died within 1-year follow-up. In the multivariate analysis, rapid NAT2 acetylator status increased the risk of death when compared against NAT2 intermediate acetylator (adjusted hazard ratio [aHR]: 1.83, 95\% CI: $1.15-2.91)$. The effect of $N A T 2$ rapid acetylator on deaths is more significant in HIV positive TB patients (aHR 2.68, 95\% CI:1.14-6.26). The risk factors associated with death was different among the NAT2 acetylators. In NAT2 rapid acetylator group, elderly people, HIV positive, past TB history and smoking status was increased the risk of death.

The NAT2 rapid acetylator is related to the mortality during TB treatment. The inadequate treatment with the doses of standard regimens caused by NAT2 rapid acetylator might increase the risk of death in TB patients, these NAT2 pharmacogenetic risks are interacting with other clinical risk factors, which is depended on the acetylator status.

\subsection{3} doi: $10.15789 / 2220-7619-2018-4-6.33$

\section{CLICHES AND DOGMAS IN MOLECULAR TUBERCULOSIS RESEARCH}

\section{Mokrousov}

\section{St. Petersburg Pasteur Institute, St. Petersburg, Russia}

I will present a personal critical view on some hot issues of the molecular epidemiology of tuberculosis, in particular, regarding an uncritical use of some well known online tools and resources. Invaluable for establishing terminology and classification in molecular epidemiological studies of Mycobacterium tuberculosis, they are limited by our insufficient knowledge of genome evolution and uncritical perception of their indications. This is exemplified by partly inadequate (sub)clade assignment due to imperfect decision rules, and misleading methodological approach when scientifically unsound phylogenies are built from spoligotyping data.

To begin with, I propose the following definitions. First, "molecular mythology" that relies on minimal array of references that conveniently support long-lasting clichés. Second, "molecular iconography" that relies on dogmatic perception of the current knowledge when online databases are uncritically regarded as ideal icons. Finally, I introduce the term "click science". In contrast to the fascinating and sophisticated click languages, "click science" relies on uncritical and simplified perception of knowledge and a dogmatic, iconographic view of indications provided by increasingly convenient online tools and databases. For example, spolTools is an example of the click phylogenetics when a plethora of statistics is generated in few clicks but their exploration is minimal. In its turn, click systematics is exemplified by SITVIT's (i) reader-unfriendly huge tables with different possible percentages and (ii) easy to read but partly inadequate (sub)clade labels.

Labels are convenient for classification, but should be revisited in the context of modern knowledge. The "we have been taught this way" approach reflects the mentality of a conservative teacher rather than a creative researcher. As Heidegger once said, "knowledge does not think"; indeed why think when it already knows? As far as science is concerned, this quotation from Henry Gee's "The accidental species" is much more appropriate: "Science is about neither Facts nor Truth, but the quantification of doubt".

Below are examples of some clichés pertaining in molecular epidemiology of tuberculosis.

Firstly, pathogenic properties of the Beijing genotype are traditionally listed as increased virulence, association 